\title{
Lymph node metastasis risk factors and applicability of endoscopic submucosal dissection in mixed-type early gastric cancer in Chinese patients
}

\author{
Pengwei Liu ${ }^{1 \#}$, Lin $\mathrm{Li}^{1 \#}$, Jing Wang ${ }^{1}$, Hong Song ${ }^{2}$, Chiyi He ${ }^{1}$ \\ ${ }^{1}$ Departments of Gastroenterology, The First Affiliated Hospital of Wannan Medical College, Wuhu, China; ${ }^{2}$ Departments of Gastrointestinal \\ Pathology, The First Affiliated Hospital of Wannan Medical College, Wuhu, China \\ Contributions: (I) Conception and design: All authors; (II) Administrative support: All authors; (III) Provision of study materials or patients: All \\ authors; (IV) Collection and assembly of data: All authors; (V) Data analysis and interpretation: All authors; (VI) Manuscript writing: All authors; (VII) \\ Final approval of manuscript: All authors. \\ \#These authors contributed equally to this work. \\ Correspondence to: Chiyi He, MD. Departments of Gastroenterology, The First Affiliated Hospital of Wannan Medical College, Wuhu 241001, China. \\ Email: hechiyi11@163.com.
}

\begin{abstract}
Background: To analyze the risk factors of lymph node metastasis (LNM) of mixed-type early gastric cancer (EGC), and to explore whether endoscopic submucosal dissection (ESD) is applicable to mixed-type EGC in Chinese patients.

Methods: A total of 812 EGC patients were included. We classified the lesions into four types: pure moderately differentiated (PMD) adenocarcinoma, mixed predominantly moderately differentiated (MMD) type, mixed predominantly poorly differentiated (MPD) type, and pure poorly differentiated (PPD) adenocarcinoma. LNM risk factors in EGC were evaluated by univariate and multivariate analyses, and the feasibility of ESD in mixed-type EGC was estimated.

Results: The LNM rate in mixed-type EGC was 24.7\% (68/275). Tumor size [odds ratio $(\mathrm{OR})=1.419$, $\mathrm{P}=0.008$, $\mathrm{MPD}(\mathrm{OR}=3.278, \mathrm{P}=0.002)$, submucosal invasion $\geq 500 \mu \mathrm{m}(\mathrm{OR}=5.059, \mathrm{P}=0.002)$, and lymphovascular invasion (LVI) ( $\mathrm{OR}=5.836, \mathrm{P}<0.001)$ were independent predictors of $\mathrm{LNM}$ in mixed-type EGC. LNM was more common in MMD patients than in PMD patients who met the expanded indications for ESD of differentiated EGC (0.0\% vs. $7.84 \%, \mathrm{P}=0.005)$.

Conclusions: Tumor size, histology, invasion depth, and LVI are independent risk factors for LNM in mixed-type EGC. The absolute indications for ESD are applicable to MMD, and the feasibility of the expanded indications for ESD in MMD and MPD requires further investigation in Chinese patients.
\end{abstract}

Keywords: Early gastric cancer (EGC); mixed-type; lymph node metastasis (LNM); endoscopic submucosal dissection (ESD)

Submitted Jul 07, 2021. Accepted for publication Aug 19, 2021.

doi: $10.21037 /$ jgo-21-462

View this article at: https://dx.doi.org/10.21037/jgo-21-462

\section{Introduction}

Gastric cancer is one of the most common malignancies and is a major cause of cancer-related deaths worldwide (1). Early gastric cancer (EGC) is defined as cancer tissue confined to the gastric mucosa or submucosa, regardless of lymph node metastasis (LNM) (2). Recently, with the development of endoscopic techniques, endoscopic submucosal dissection (ESD) has been widely applied in EGC without LNM. Therefore, the LNM rate of EGC is the main factor determining whether ESD can be performed. ESD is developed from endoscopic mucosal resection (EMR). The lesions located in the mucosal layer are separated from the 


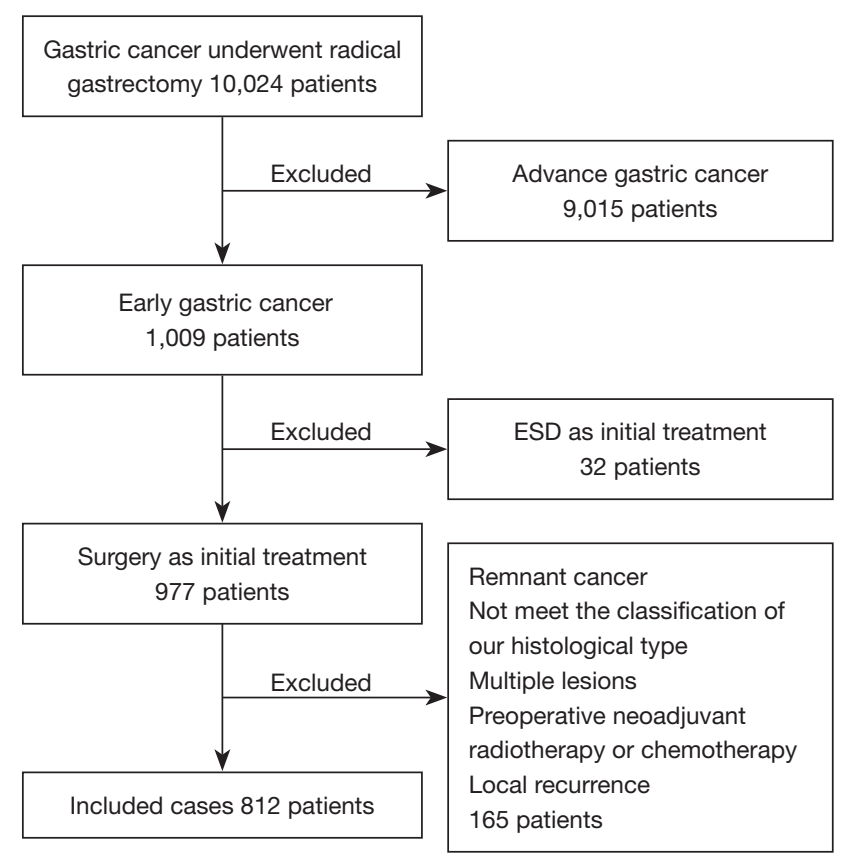

Figure 1 Flowchart of patient inclusion and exclusion. ESD, endoscopic submucosal dissection.

base layer and removed completely by means of electric knife and other separation tools. The use of ESD in EGC surgery can achieve the effect of radical resection of tumor and effectively reduce postoperative tumor residue and recurrence. On the basis of the Japanese Classification of Gastric Carcinoma (JCGC), gastric cancer can be divided into differentiated and undifferentiated types, and tumors with a mixture of the two types are classified in accordance with the major histological type (2). Recent studies have proven that the risk of LNM is negligible in differentiated and undifferentiated tumors that meet certain criteria. Thus, the ESD indications are expanding to EGC with larger sizes, with ulcers or superficial submucosal invasion (3-5). However, the risk of LNM in mixed EGC remains controversial, and no agreement has yet been reached on the ESD indications for mixed tumors. Some studies reported that the absolute or expanded indications of ESD for pure differentiated or undifferentiated EGC could be applicable to mixed predominantly differentiated- or undifferentiatedtype EGC (6,7). Several recent studies have reported that histological mixed-type EGC has a greater risk of LNM than the undifferentiated type (8-10), and ESD might not be suitable for mixed-type tumors. In addition, mixed gastric cancer contains heterogeneous types of cells which may possess different biological behaviors. Although there have been some studies on the LNM of histologically mixed-type EGCs, the behavior of moderately-poorly differentiated adenocarcinoma, which is the most common mixedtype histology of EGC, has not been reported separately. Furthermore, no study has addressed the differences in LNM between mixed predominantly moderately differentiated (MMD) type and pure moderately differentiated (PMD) adenocarcinoma, as well as mixed predominantly poorly differentiated (MPD) type and pure poorly differentiated (PPD) adenocarcinomas. The objective of the present study is to explore the clinicopathological characteristics of mixed-type EGC, and to investigate the ESD indications for MMD and MPD in Chinese patients. The pathological types involved in this paper are relatively comprehensive, including PMD, MMD, MPD and PPD. The conclusions are convincing.

We present the following article in accordance with the STROBE reporting checklist (available at https://dx.doi. org/10.21037/jgo-21-462).

\section{Methods}

We identified 10,024 consecutive patients who underwent radical gastrectomy and were diagnosed with gastric cancer according to postoperative pathology between March 2012 and July 2020 at the First Affiliated Hospital of Wannan Medical College. A total of $812(8.1 \%)$ patients who were diagnosed with EGC by postoperative pathological examination were retrospectively analyzed (Figure 1).

The exclusion criteria were as follows: (I) remnant gastric cancer; (II) preoperative neoadjuvant radiotherapy or chemotherapy; (III) less than 15 lymph node dissections; (IV) preoperative endoscopic treatment; (V) local recurrence; (VI) multiple synchronous EGC.

All procedures performed in this study involving human participants were in accordance with the Declaration of Helsinki (as revised in 2013). The study was approved by ethics board of First Affiliated Hospital of Wannan Medical College (No.: 2012-004). Individual consent for this retrospective analysis was waived.

Clinicopathological factors were analyzed, including gender, age, location, tumor size, depth of invasion, ulceration, macroscopic type, lymphovascular invasion (LVI), and LNM. On the basis of the treatment guidelines of the Japanese Gastric Cancer Association (JGCA) (2), the location of gastric cancer was separated into upper, middle, and lower thirds. Based on the Paris endoscopic classification of superficial tumor lesions, we defined the macroscopic types 

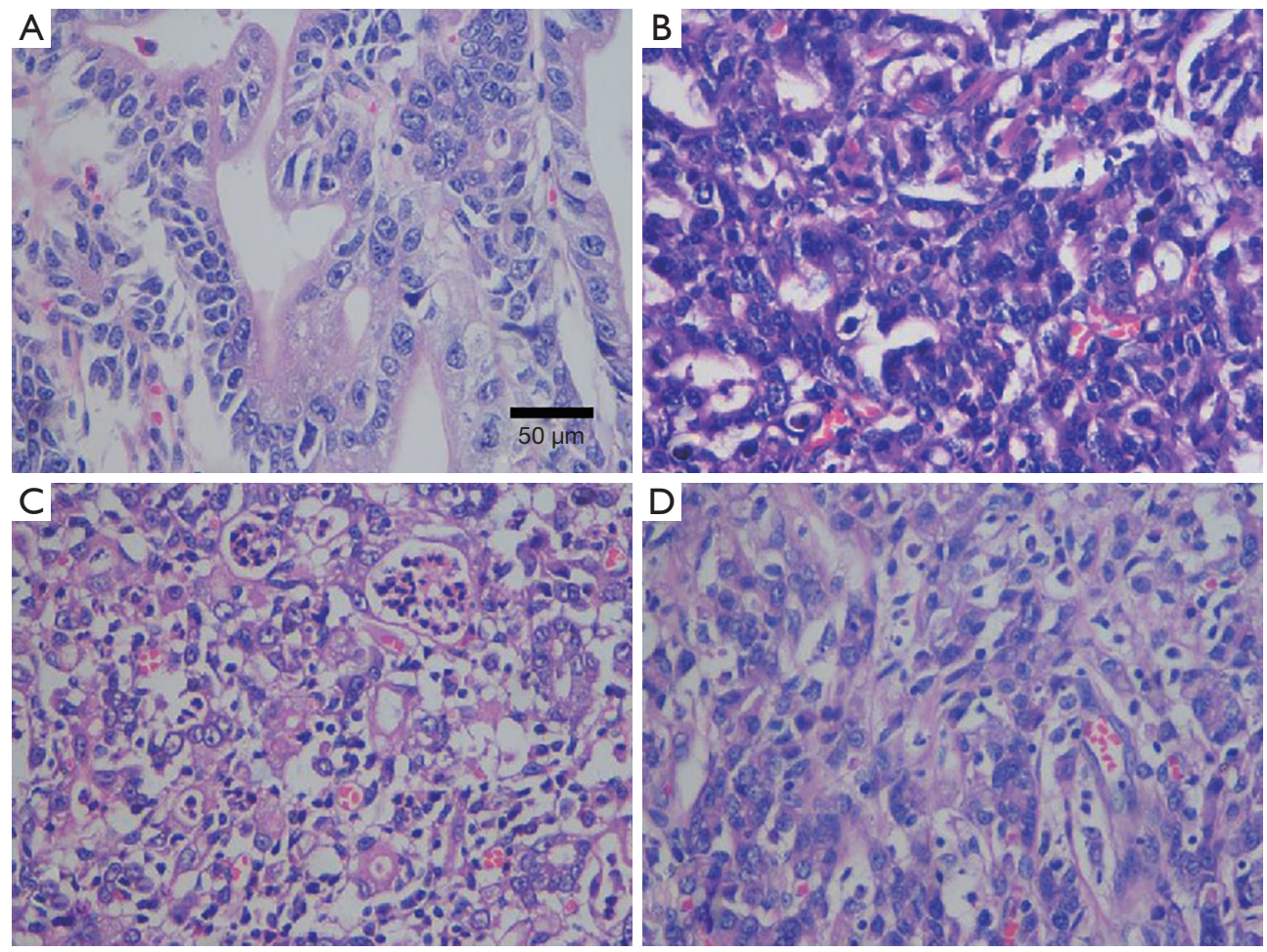

Figure 2 The four different types of EGC. (A) PMD adenocarcinoma, it was composed of moderately differentiated type only; (B) MMD type adenocarcinoma, the moderately components accounting for more than 50\%; (C) MPD type adenocarcinoma, the poorly components accounting for more than 50\%; (D) PPD adenocarcinoma, it was composed of poorly differentiated type only. HE (the method of staining), ×400 (internal scale). EGC, early gastric cancer; PMD, pure moderately differentiated; MMD, mixed predominantly moderately differentiated; MPD, mixed predominantly poorly differentiated; PPD, pure poorly differentiated.

of tumors as elevated type (0-I and 0-IIa), flat type (0-IIb), and depressed type (0-IIc and 0-III) (11). We divided the depth of invasion into three different categories: $M$ (mucosa), SM1 (submucosal $<500 \mu \mathrm{m}$ ), SM2 (submucosal $\geq 500 \mu \mathrm{m}$ ). The submucosal invasion depth was measured from the lowest point of the muscularis mucosa to the deepest tumor invasion point.

All slides were reviewed to estimate the percentages of each tumor component (moderately differentiated or poorly differentiated adenocarcinoma) based on the criteria of the WHO classification. All sections per case were analyzed. Five to 10 high magnification visual fields $(x 400)$ were randomly observed in each section. The four different types of EGC. PMD adenocarcinoma, it was composed of moderately differentiated type only (Figure $2 A$ ); MMD type adenocarcinoma, the moderately components accounting for more than $50 \%$ (Figure 2B); MPD type adenocarcinoma, the poorly components accounting for more than $50 \%$ (Figure 2C); PPD adenocarcinoma, it was composed of poorly differentiated type only (Figure $2 D$ ). HE (the method of staining), $\times 400$ (internal scale). The results were comprehensively judged according to the proportion of tumor components. We classified the lesions into four categories based on the major histopathological components: PMD type (moderately differentiated adenocarcinoma accounting for more than $80 \%$ ), MMD type (moderately differentiated components accounting for more than $50 \%$ ), MPD type (poorly differentiated adenocarcinoma components accounting for more than $50 \%$ ), and PPD type (poorly differentiated adenocarcinoma accounting for more than $80 \%$ ) (Figure 2). Hematoxylin and eosin staining was used to diagnose LNM. LVI was deemed to be present when tumor cells were found in the tubular space lined by endothelial cells or in the structure of the vessel wall.

\section{Statistical analysis}

Statistical analyses were performed with the software SPSS 
26.0 (SPSS, version 26.0, Chicago, IL, USA). The chisquared test or Fisher's exact test was used to compare the categorical variables. Student's $t$-test was performed to compare the two groups of continuous variables. The multivariate logistic regression model was applied to analyze the independent risk factors for LNM in EGC. $\mathrm{P}<0.05$ was considered statistically significant.

\section{Results}

\section{Clinicopatbological characteristics}

Figure 1 showed a flow diagram of this study. A total of 812 EGC patients who met the criteria were involved in this study, and the clinicopathological characteristics are given in Table 1. Overall, 571 (70.3\%) were male and 241 (29.7\%) were female, with an average age of 60.9 (range, 25-84) years. About half of the tumors (51.1\%) were intramucosal cancer. The mean lesion size was $21 \mathrm{~mm}$. Patients were divided into four groups according to the histopathology: PMD, 401 (49.4\%); MMD, 134 (16.5\%); MPD, 141 (17.4\%); and PPD, 136 (16.7\%). The total LVI rate was $10.1 \%$, and LNM was present in 127 of the 812 patients (15.6\%).

\section{Univariate and multivariate analysis of LNM risk factors in EGC}

The univariate analysis showed that LNM in EGC was significantly associated with the depth of invasion $(\mathrm{P}<0.001)$, tumor size $(\mathrm{P}<0.000)$, histological type $(\mathrm{P}<0.001)$, presence of ulceration $(\mathrm{P}=0.002)$, and LVI $(\mathrm{P}<0.001)$ (Table 1). The multivariate logistic regression analysis showed that tumor size [odds ratio $(\mathrm{OR})=1.381,95 \%$ confidence interval $(\mathrm{CI})$ : $1.168-1.634, \mathrm{P}<0.001$ ], mixed type of MPD $(\mathrm{OR}=7.412$, 95\% CI: $3.908-14.059, \mathrm{P}<0.001)$, submucosal invasion $(\geq 500 \mu \mathrm{m})(\mathrm{OR}=8.565,95 \% \mathrm{CI}: 4.558-16.094, \mathrm{P}<0.001)$, and LVI (OR =9.297, 95\% CI: 5.141-16.814, $\mathrm{P}<0.001)$ were independent risk factors for LNM in EGC (Table 2).

\section{Subgroup analysis of LNM risk factors}

Based on the results of the aforementioned analyses, the multivariate analysis showed that histological type was an independent predictor of LNM in EGC. Furthermore, univariate analysis and multivariate logistic regression were performed to assess the risk factors of LNM in mixedtype EGC (MMP and MPD groups). The total LNM rate was $24.7 \%$ (68/275). Table 3 shows that the LNM rate was significantly associated with the macroscopic type $(\mathrm{P}=0.036)$, depth of invasion $(\mathrm{P}<0.001)$, tumor size $(\mathrm{P}<0.001)$, histological type $(\mathrm{P}<0.001)$, and LVI $(\mathrm{P}<0.001)$. Multivariate analysis found that tumor size (OR $=1.419,95 \%$ CI: $1.096-$ 1.837, $\mathrm{P}=0.008)$, mixed type of MPD (OR $=3.278,95 \% \mathrm{CI}$ : 3.529-12.288, $\mathrm{P}=0.002)$, submucosal invasion $(\geq 500 \mu \mathrm{m})$ (OR $=5.059$, 95\% CI: 1.818-14.279, $\mathrm{P}=0.002)$, and LVI (OR =5.836, 95\% CI: 2.631-12.947, $\mathrm{P}<0.001)$ were independent risk factors for LNM in mixed-type EGC (Table 4).

\section{Comparison of LNM rates in EGC according to indications for $E S D$}

Further analysis revealed that no LNM metastasis was found in patients who met the absolute indications for ESD of differentiated EGC in the PMD (0/156) and MMD (0/45) groups. However, the LNM rate was higher in MMD patients than in PMD patients $(0.0 \%$ vs. $7.84 \%, \mathrm{P}=0.005)$ who met the expanded indications for ESD of differentiated EGC: (I) differentiated mucosal tumors with no ulcerative findings and LVI, and of any size; (II) differentiated mucosal tumors, $\leq 30 \mathrm{~mm}$ in size, with ulcerative findings and without LVI; (III) differentiated tumors with SM1 $(<500 \mu \mathrm{m})$ invasion, without LVI, and $\leq 30 \mathrm{~mm}$ in size (Table 5). As shown in Table 6, LNM occurred more frequently in MPD patients than in PPD patients who met the expanded indications for ESD in undifferentiated EGC, while there were no significant differences between the two groups (0.0\% vs. $4.76 \%, \mathrm{P}=0.373$ ).

\section{Discussion}

LNM is a key factor in selecting the suitable treatment modality for EGC (4). Histological differentiation is an independent risk factor for LNM and probably assists in deciding the appropriate treatment strategy for EGC (12-14). ESD is widely used in the treatment of submucosal lesions of upper digestive tract. Endoscopic therapy can effectively treat EGC and has gradually become the first-line treatment of EGC with the increasing maturity of ESD technology. The ability of life and quality of life of patients with EGC have been significantly improved. However, gastric cancer tissues usually have a mixed-type histology with both undifferentiated and differentiated components. Studies have proven that mixed-type EGC is more aggressive than pure-type EGC $(15,16)$, while some studies have shown that ESD under the expanded criteria could be applied to certain mixed-type EGCs $(17,18)$. However, the studies did 
Table 1 Univariate analysis of factors for LNM in EGC

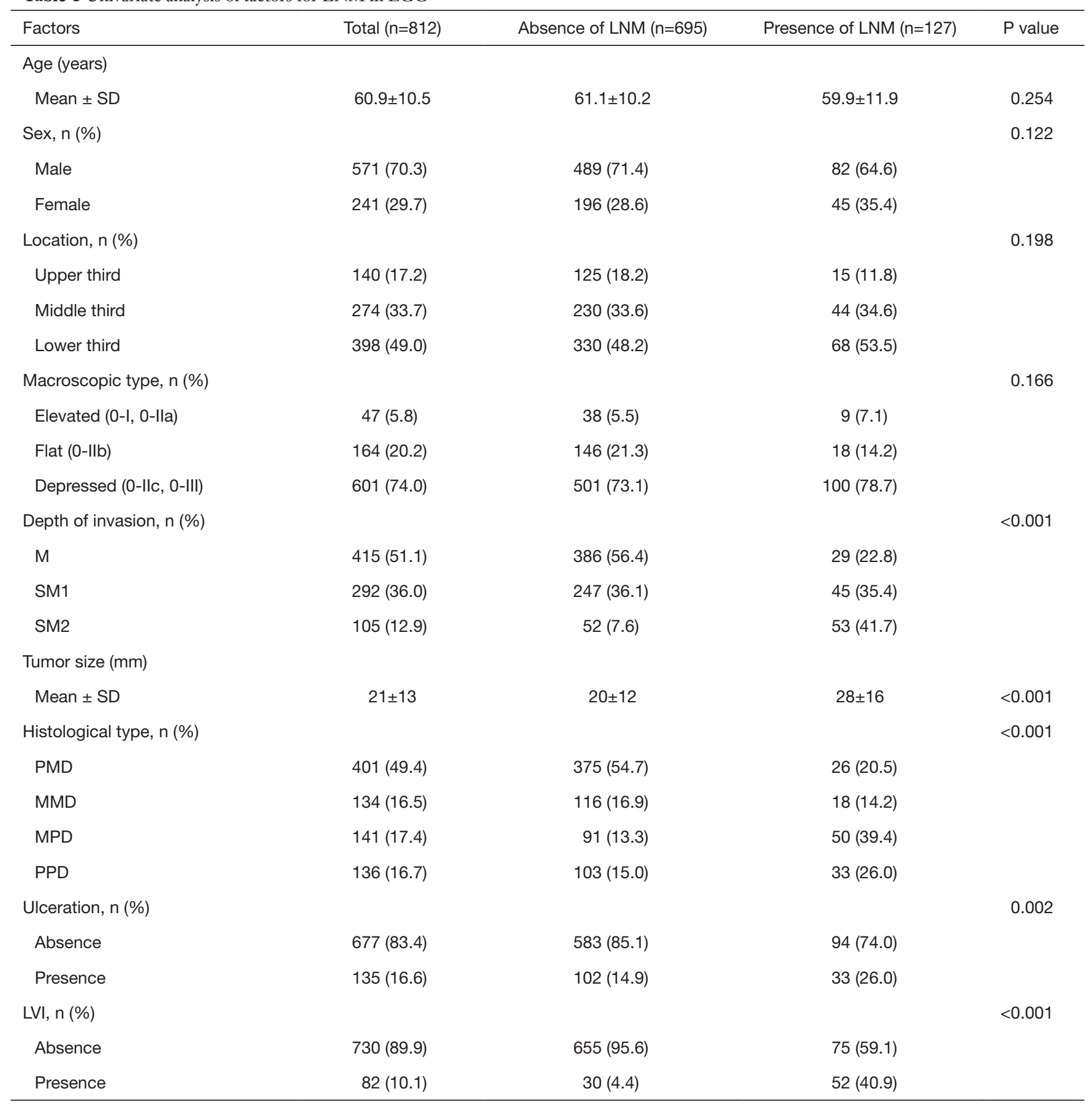

LNM, lymph node metastasis; EGC, early gastric cancer; M, mucosa; SM1, submucosal $<500 \mu m$; SM2, submucosal $\geq 500 \mu m$; PMD, pure moderately differentiated adenocarcinoma; MMD, mixed predominantly moderately differentiated adenocarcinoma; MPD, mixed predominantly poorly differentiated adenocarcinoma; PPD, pure poorly differentiated adenocarcinoma; LVI, lymphovascular invasion. 
Table 2 Multivariate analysis of factors for LNM in EGC

\begin{tabular}{lccc}
\hline Variables & OR & $95 \%$ Cl & P value \\
\hline Tumor size & 1.381 & $1.168-1.634$ & $<.001$ \\
Histology & 1 & & $<0.001$ \\
PMD & 2.307 & $1.094-4.866$ & 0.028 \\
MMD & 7.412 & $3.908-14.059$ & $<0.001$ \\
MPD & 4.329 & $2.247-8.341$ & $<0.001$ \\
PPD & & & $<0.001$ \\
Depth of invasion & 1 & & 0.165 \\
M & 1.487 & $0.849-2.607$ & $<0.001$ \\
SM1 & 8.565 & $4.558-16.094$ & \\
SM2 & & & $<0.001$ \\
LVI & 1 & $5.141-16.814$ & \\
Absence & 9.297 &
\end{tabular}

LNM, Iymph node metastasis; EGC, early gastric cancer; OR, odds ratio; Cl, confidence interval; PMD, pure moderately differentiated adenocarcinoma; MMD, mixed predominantly moderately differentiated adenocarcinoma; MPD, mixed predominantly poorly differentiated adenocarcinoma; PPD, pure poorly differentiated adenocarcinoma; M, mucosa; SM1, submucosal $<500 \mu \mathrm{m}$; SM2, submucosal $\geq 500 \mu$ m; LVI, lymphovascular invasion.

not distinguish the various components of differentiated or undifferentiated EGC, which may have individual biological behaviors. Our study analyzed the clinicopathological features and LNM risk factors of the most common mixed-type EGCs of moderately-poorly differentiated adenocarcinoma, and clarified the applicability of ESD for this mixed-type EGC in Chinese patients.

Our study demonstrated that the total LNM rate was $15.6 \%$ in EGC, and clinicopathological features submucosal invasion, tumor size, and LVI were independent risk factors for LNM, which was in accordance with several previous studies (19-21). As the multivariate analysis revealed that histological type was one of the independent predictors for LNM in EGC, we further performed a subgroup analysis of clinicopathological features and LNM risk factors in mixed-type EGC. Parallel with a recent study (22), we found that LNM occurred more often in mixed-type EGC (24.7\%) than in EGC which included only the two pure types (11.0\%).

Tumor size has been recognized as an independent risk factor for LNM in EGC in some studies $(19,23,24)$, and we gained the same conclusion in mixed-type EGC. Our data showed that MPD had a higher LNM rate than MMD which was in accordance with a recent study (25).
This indicated that the higher the proportion of poorly differentiated components, the higher the risk of LNM in mixed-type EGC. Chu et al. found that the deeper the tumor invasion, the higher the LNM rate (24). In line with this study, we also found that depth of invasion was an independent risk factor for LNM in mixed-type EGC, and patients with SM2 invasion tended to have a higher LNM rate compared to patients with mucosa or SM1 invasion $(\mathrm{P}=0.008)$. Regarding LVI, many studies have shown that LVI is an extremely important independent factor for LNM in EGC, and our research also proved that mixed-type EGC with LVI had more aggressive behavior than in patients without LVI (21).

We further explored whether the ESD indications for EGC are applicable to MMD and MPD. Our study demonstrated that no LNM occurred in patients who met the absolute indications for ESD of differentiated EGC in both the PMD (0/156) and MMD (0/45) groups. The LNM rate was higher in MMD than in PMD patients who met the expanded indications $(7.84 \%$ vs. $0.0 \%, \mathrm{P}=0.005)$. A study from Japan observed that no LNM occurred in differentiated EGC patients who met the expanded indications for ESD (26), while Kang et al. showed that the LNM rate of differentiated EGC was 2.4\% among Korean 
Table 3 Univariate analysis of factors for LNM in mixed-type EGC

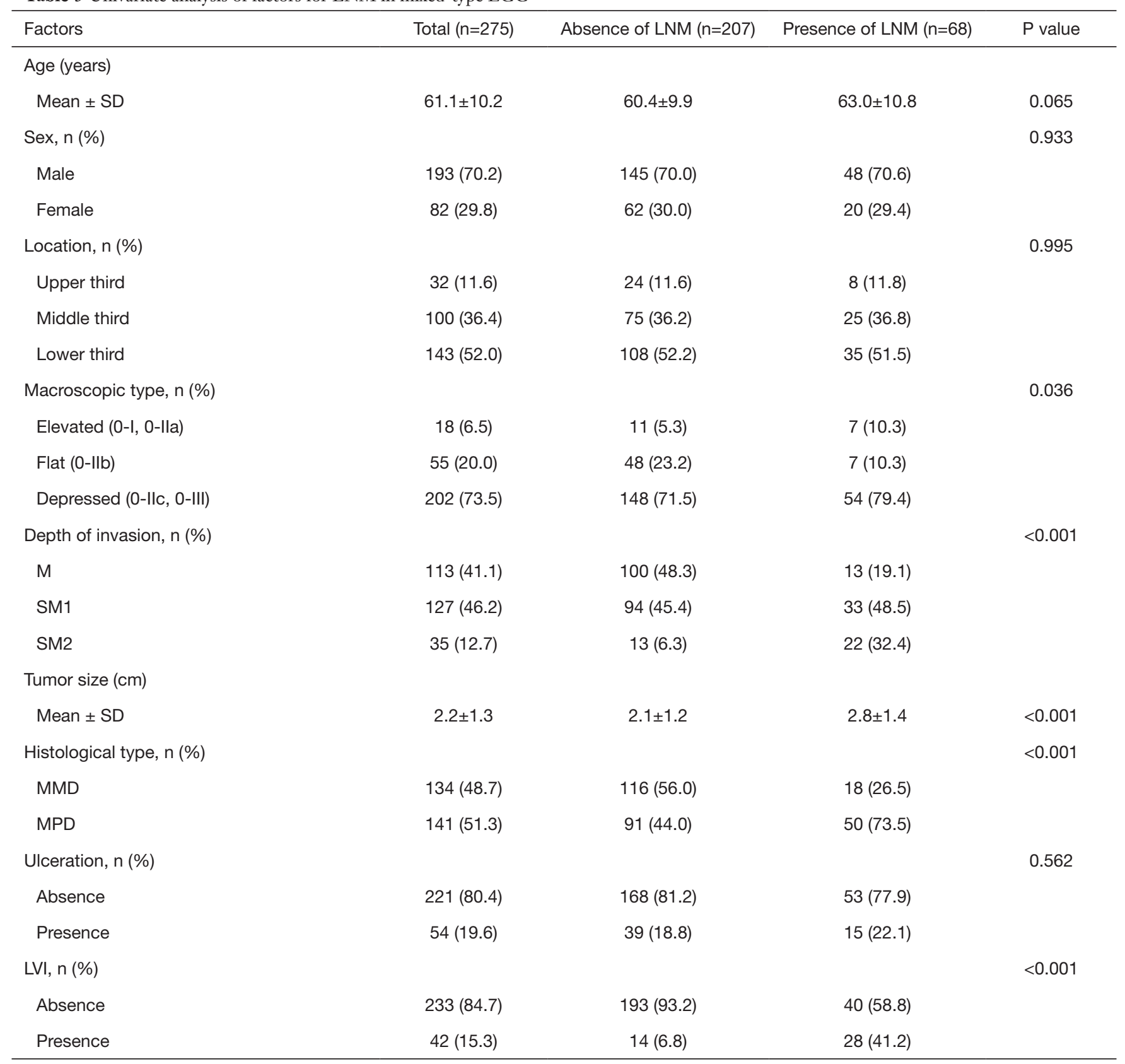

LNM, lymph node metastasis; EGC, early gastric cancer; M, mucosa; SM1, submucosal <500 $\mu$; $S M 2$, submucosal $\geq 500 \mu m$; MMD, mixed predominantly moderately differentiated adenocarcinoma; MPD, mixed predominantly poorly differentiated adenocarcinoma; LVI, lymphovascular invasion.

patients who met the expanded indications (27). Thus, we deemed that the ESD indications might be feasible for MMD patients who met the absolute indications, while they might not be suitable for MMD patients who met the expanded indications for differentiated EGC. Meanwhile, we found that the LNM rate of MPD patients who met the expanded indications of undifferentiated EGC was higher than that of PPD patients $(4.76 \%$ vs. $0.0 \%)$, though there was no significant difference in LNM between MPD and PPD $(\mathrm{P}=0.373)$. We considered that this result 
Table 4 Multivariate analysis of factors for LNM in mixed-type EGC

\begin{tabular}{|c|c|c|c|}
\hline Variables & OR & $95 \% \mathrm{Cl}$ & $P$ value \\
\hline Histology & & & 0.002 \\
\hline MMD & 1 & & \\
\hline MPD & 3.278 & $3.529-12.288$ & \\
\hline M & 1 & & \\
\hline SM1 & 1.896 & $0.882-4.073$ & 0.101 \\
\hline SM2 & 5.059 & $1.818-14.279$ & 0.002 \\
\hline LVI & & & $<0.001$ \\
\hline
\end{tabular}

LNM, lymph node metastasis; EGC, early gastric cancer; OR, odds ratio; Cl, confidence interval; MMD, mixed predominantly moderately differentiated adenocarcinoma; MPD, mixed predominantly poorly differentiated adenocarcinoma; M, mucosa; SM1, submucosal $<500 \mu \mathrm{m}$; SM2, submucosal $\geq 500 \mu \mathrm{m}$; LVI, lymphovascular invasion.

Table 5 Comparison of LNM between PMD and MMD according to the ESD indications for differentiated EGC

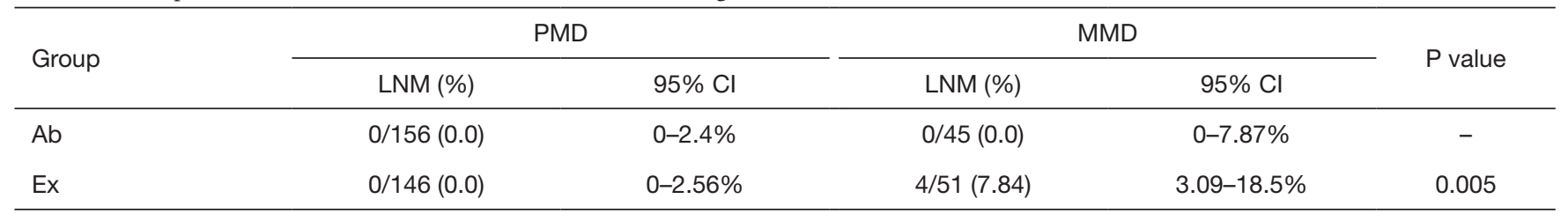

LNM, lymph node metastasis; PMD, pure moderately differentiated adenocarcinoma; MMD, mixed predominantly moderately differentiated adenocarcinoma; ESD, endoscopic submucosal dissection; EGC, early gastric cancer; $\mathrm{Cl}$, confidence interval; Ab, absolute indications for ESD of differentiated EGC; Ex, expanded indications for ESD of differentiated EGC.

Table 6 Comparison of LNM between PPD and MPD according to the ESD indications for undifferentiated EGC

\begin{tabular}{|c|c|c|c|c|c|}
\hline Group & \multicolumn{2}{|c|}{ PPD } & \multicolumn{2}{|c|}{ MPD } & $P$ value \\
\hline Ex & $0 / 37(0.0)$ & $0-9.41 \%$ & $1 / 21(4.76)$ & $0.85-22.67 \%$ & 0.373 \\
\hline
\end{tabular}

LNM, lymph node metastasis; PPD, pure poorly differentiated adenocarcinoma; MPD, mixed predominantly poorly differentiated adenocarcinoma; ESD, endoscopic submucosal dissection; EGC, early gastric cancer; Cl, confidence interval; Ex, expanded indications for ESD of undifferentiated EGC.

might be associated with the small sample size. Therefore, the feasibility of expanded indications for ESD in MPD requires further investigation in Chinese patients.

Tumor size, histology, invasion depth, and LVI are independent risk factors for LNM in mixed-type EGC. The absolute indications for ESD are applicable to MMD, and the feasibility of the expanded indications for ESD in MMD and MPD requires further investigation in Chinese patients. The limitations of this study include its retrospective and single-center nature. Another limitation of the study was that the sections of the surgically resected specimens were prepared at $5-\mathrm{mm}$ intervals for pathological diagnosis, while ESD specimens were prepared at 2-mm intervals. Prospective studies regarding the long-term prognosis of 
post-ESD patients with mixed-type EGC are necessary to establish the standard treatment. However, this study includes continuity of clinical cases treated in the third-class hospital of Wannan region of Anhui Province over a period of 10 years ensuring a meaningful investigation.

In conclusion, our results demonstrated that it was feasible to use the current absolute indications for ESD in cases of MMD, and the feasibility of the expanded indications for ESD in MMD and MPD requires further investigation in Chinese patients. We recommend quantitative evaluation and careful consideration when deciding whether mixed-type EGC patients need additional surgery after ESD.

\section{Acknowledgments}

Funding: This work was supported by the Anhui Province Key Research and Development Program Project (grant number: No. 201904a07020028).

\section{Footnote}

Reporting Checklist: The authors have completed the STROBE reporting checklist. Available at https://dx.doi. org/10.21037/jgo-21-462

Data Sharing Statement: Available at https://dx.doi. org/10.21037/jgo-21-462

Conflicts of Interest: All authors have completed the ICMJE uniform disclosure form (available at https://dx.doi. org/10.21037/jgo-21-462). The authors have no conflicts of interest to declare.

Ethical Statement: The authors are accountable for all aspects of the work in ensuring that questions related to the accuracy or integrity of any part of the work are appropriately investigated and resolved. All procedures performed in this study involving human participants were in accordance with the Declaration of Helsinki (as revised in 2013). The study was approved by ethics board of First Affiliated Hospital of Wannan Medical College (No.: 2012004). Individual consent for this retrospective analysis was waived.

Open Access Statement: This is an Open Access article distributed in accordance with the Creative Commons Attribution-NonCommercial-NoDerivs 4.0 International
License (CC BY-NC-ND 4.0), which permits the noncommercial replication and distribution of the article with the strict proviso that no changes or edits are made and the original work is properly cited (including links to both the formal publication through the relevant DOI and the license). See: https://creativecommons.org/licenses/by-nc-nd/4.0/.

\section{References}

1. Balakrishnan M, George R, Sharma A, et al. Changing trends in stomach cancer throughout the world. Curr Gastroenterol Rep 2017;19:36.

2. Japanese Gastric Cancer Association. Japanese gastric cancer treatment guidelines 2018 (5th edition). Gastric Cancer 2021;24:1-21.

3. Gotoda T, Yamamoto H, Soetikno RM. Endoscopic submucosal dissection of early gastric cancer. J Gastroenterol 2006;41:929-42.

4. Hirasawa T, Gotoda T, Miyata S, et al. Incidence of lymph node metastasis and the feasibility of endoscopic resection for undifferentiated-type early gastric cancer. Gastric Cancer 2009;12:148-52.

5. Abdelfatah MM, Barakat $M$, Lee $H$, et al. The incidence of lymph node metastasis in early gastric cancer according to the expanded criteria in comparison with the absolute criteria of the Japanese Gastric Cancer Association: a systematic review of the literature and meta-analysis. Gastrointest Endosc 2018;87:338-47.

6. Takizawa K, Ono H, Kakushima N, et al. Risk of lymph node metastases from intramucosal gastric cancer in relation to histological types: how to manage the mixed histological type for endoscopic submucosal dissection. Gastric Cancer 2013;16:531-6.

7. Min BH, Kim KM, Park CK, et al. Outcomes of endoscopic submucosal dissection for differentiated-type early gastric cancer with histological heterogeneity. Gastric Cancer 2015;18:618-26.

8. Miyamae M, Komatsu S, Ichikawa D, et al. Histological mixed-type as an independent risk factor for nodal metastasis in submucosal gastric cancer. Tumour Biol 2016;37:709-14.

9. Mikami K, Hirano Y, Futami K, et al. Expansion of lymph node metastasis in mixed-type submucosal invasive gastric cancer. Asian J Surg 2018;41:462-6.

10. Hwang CS, Ahn S, Lee BE, et al. Risk of lymph node metastasis in mixed-type early gastric cancer determined by the extent of the poorly differentiated component. World J Gastroenterol 2016;22:4020-6. 
11. The Paris endoscopic classification of superficial neoplastic lesions: esophagus, stomach, and colon: November 30 to December 1, 2002. Gastrointest Endosc 2003;58:S3-43.

12. Pyo JH, Lee H, Min BH, et al. Long-term outcome of endoscopic resection vs. surgery for early gastric cancer: a non-inferiority-matched cohort study. Am J Gastroenterol 2016;111:240-9.

13. Komatsu S, Ichikawa D, Miyamae M, et al. Histological mixed-type as an independent prognostic factor in stage I gastric carcinoma. World J Gastroenterol 2015;21:549-55.

14. Zheng H, Takahashi H, Murai Y, et al. Pathobiological characteristics of intestinal and diffuse-type gastric carcinoma in Japan: an immunostaining study on the tissue microarray. J Clin Pathol 2007;60:273-7.

15. Zheng HC, Li XH, Hara T, et al. Mixed-type gastric carcinomas exhibit more aggressive features and indicate the histogenesis of carcinomas. Virchows Arch 2008;452:525-34.

16. Pyo JH, Lee H, Min BH, et al. Early gastric cancer with a mixed-type Lauren classification is more aggressive and exhibits greater lymph node metastasis. J Gastroenterol 2017;52:594-601.

17. Yoon HJ, Kim YH, Kim JH, et al. Are new criteria for mixed histology necessary for endoscopic resection in early gastric cancer? Pathol Res Pract 2016;212:410-4.

18. Zhong Q, Sun Q, Xu GF, et al. Differential analysis of lymph node metastasis in histological mixed-type early gastric carcinoma in the mucosa and submucosa. World J Gastroenterol 2018;24:87-95.

19. Du MZ, Gan WJ, Yu J, et al. Risk factors of lymph node metastasis in 734 early gastric carcinoma radical resections in a Chinese population. J Dig Dis 2018;19:586-95.

20. Zhang D, Li Q, Yang L, et al. Analysis of clinicopathologic

Cite this article as: Liu P, Li L, Wang J, Song H, He C. Lymph node metastasis risk factors and applicability of endoscopic submucosal dissection in mixed-type early gastric cancer in Chinese patients. J Gastrointest Oncol 2021;12(4):1444-1453. doi: 10.21037/jgo-21-462 factors affecting formation of lymphovascular invasion in 1260 patients with gastric cancer. Chinese Journal of Digestive Surgery 2017;16: 269-74.

21. Jiao J, Guo P, Hu S, et al. Laparoscopic gastrectomy for early gastric cancer and the risk factors of lymph node metastasis. J Minim Access Surg 2019. [Epub ahead of print]. doi: 10.4103/jmas.JMAS_296_18.

22. Hu Q, Dekusaah R, Cao S, et al. Risk factors of lymph node metastasis in patients with early pure and mixed signet ring cell gastric carcinomas. J Cancer 2019;10:112431.

23. Chen L, Wang YH, Cheng YQ, et al. Risk factors of lymph node metastasis in 1620 early gastric carcinoma radical resections in Jiangsu Province in China: a multicenter clinicopathological study. J Dig Dis 2017;18:556-65.

24. Chu YN, Yu YN, Jing X, et al. Feasibility of endoscopic treatment and predictors of lymph node metastasis in early gastric cancer. World J Gastroenterol 2019;25:5344-55.

25. Lai JF, Xu WN, Noh SH, et al. Effect of World Health Organization (WHO) histological classification on predicting lymph node metastasis and recurrence in early gastric cancer. Med Sci Monit 2016;22:3147-53.

26. Gotoda T, Yanagisawa A, Sasako M, et al. Incidence of lymph node metastasis from early gastric cancer: estimation with a large number of cases at two large centers. Gastric Cancer 2000;3:219-25.

27. Kang HJ, Kim DH, Jeon TY, et al. Lymph node metastasis from intestinal-type early gastric cancer: experience in a single institution and reassessment of the extended criteria for endoscopic submucosal dissection. Gastrointest Endosc 2010;72:508-15.

(English Language Editor: C. Betlzar) 